\title{
A 215 Statistical analysis of turbulent fluctuation transfer in boundary layer around thin edge plate by using dynamic Dynamic PIV velocity data
}

\author{
N Erkan(Univ. Tokyo), M Ishikawa(Univ. Ryukyus), \\ K Okamoto(Univ. Tokyo)
}

\begin{abstract}
Dynamic PIV (Particle Imaging Velocimetry) measurement technique was applied to the high-speed mist flow in a narrow channel with an obstacle. The boundary layer was visualized with a high-speed camera and a high repetition double-pulse laser. The square flow channel has the dimensions of $10 \times 10 \mathrm{~mm}$ and an oil and nitrogen gas mixture flows through it with Reynolds

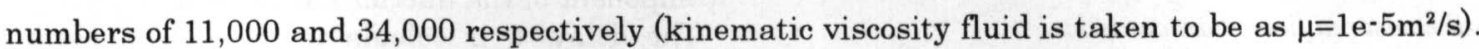
Although, the Reynolds number of 34,000 was hard to capture enough number of images, which shows vortex evolution in time, for the time resolved analysis of boundary layer, the vortex evolution at the edge of thin plate has been clearly visualized in temporal domain. Velocity fluctuation transfers velocities in the boundary layer were calculated by two-point velocity cross-correlation. Fluctuation transfer velocities were in good agreement with the some measured vortex transfer velocities.
\end{abstract}

Keywords: Two-point velocity cross correlation, dynamic PIV, mist flow

\section{Introduction}

Dynamic PIV with a high-speed camera and a high repetition laser is focused as quantitative measurement of the transient phenomenon. It is possible to expand this system to time resolution of high frequency at $1 \mathrm{kHz}, 10 \mathrm{kHz}$ and $20 \mathrm{kHz}$. It can also be widely applied as time serial measurement of PIV which has remained the two-dimensional velocity measurement. Currently, some systems using the Dynamic PIV have been developed

The mist flow is used in the industrial fields such as nuclear plant, injection systems on engines, coating system for antirust, and so on. In many applications of mist flow the speed is fast. For example, gas-liquid two-phase flows in a pipe. Mist flow indicates more than $10 \mathrm{~m} / \mathrm{s}$ in the superficial gas velocity. Also size and velocity measurements of mist are usually performed in order to improve combustion efficiency. Since previous PIV techniques obtain only the instantaneous velocity distribution, it was difficult to understand the transient phenomenon of high-speed flow.

In this paper, the target flow is the flow between fuel rods with close-packed hexagonal lattice in a next generation nuclear power reactor. The gap between rods is very narrow and $1 \mathrm{~mm}$ in minimum. Furthermore a spacer is included to fix the fuel rods in the gap. The coolant is steam with a void fraction of $99 \%$. Because of safety concerns, it is important to understand the influence of a spacer on the coolant flow characteristics in the narrow gap. Therefore, as a feasible experiment the Dynamic PIV system is applied to the high-speed mist flow around an obstacle in the narrow channel and effectiveness of the Dynamic PIV system is described.

\section{Experimental setup}

Figure 2 illustrates the schematic of the test section in this experiment. Authors used a high-speed camera (Photron APX RS, 1024x1024@3kHz) and a Nd:YLF double-pulse laser (New-wave Research Inc. Pegasus-PIV, $\lambda=523 \mu \mathrm{m}, 1 \mathrm{~mJ} @ 10 \mathrm{kHz})$. Channel size is $10 \mathrm{~mm} \times 10 \mathrm{~mm}$ in cross'section with a length of $1 \mathrm{~m}$. The obstacle with 45 -degree edge at the tip has a $2 \mathrm{~mm}$ width, sets $0.75 \mathrm{~m}$ from the downstream and is set in the center of the channel. In actual dimensions, flow field of the channel has a $1 \mathrm{~mm}$ equivalent diameter. However, it is impossible to take the images when the laser light sheet thickness becomes less than $1 \mathrm{~mm}$. Therefore, the 10 times scale-up model is used. For two flow cases, Reynolds numbers are 34,000 and 11,000 . The mist is made of olive oil suspended in Nitrogen gas obtained by using the Raskin nozzle. These oil droplets are used as tracer particles.

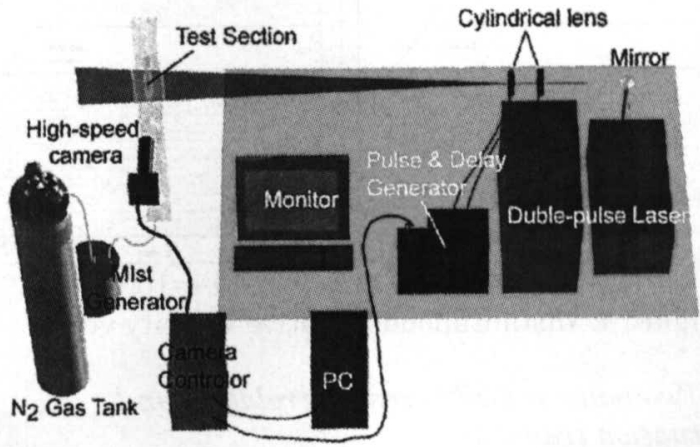

Figure 1. Experimental setup. 


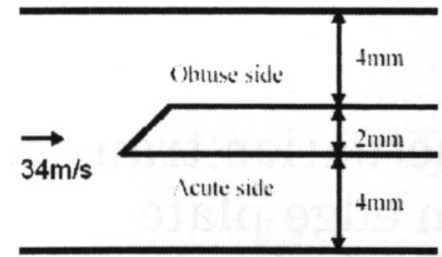

Figure 2. Schematic of test section.

The configuration of the high-speed camera was $384 \times 304$ pixels and $20 \mathrm{kHz}$ in frame rate as described in above section. The repetition rate of the double pulse laser was $10 \mathrm{kHz}$ for each rod. The frequency of this laser is $1 \mathrm{kHz}$ to $10 \mathrm{kHz}$, and energy per pulse is $10 \mathrm{~mJ}$ at $1 \mathrm{kHz}$ to $1 \mathrm{~mJ}$ at $10 \mathrm{kHz}$. The double pulse interval is $2 \mu \mathrm{s}$ using frame-straddling technique.

\section{Methods, Results and Discussion}

\subsection{General measured flow characteristics}

The velocity vector maps are calculated by using recursive PIV analysis technique. The time intervals between two-consecutive images for PIV evaluation are $2 \mu$ s and $10 \mu \mathrm{s}$ for the cases of 34,000 and 11,000 Reynolds numbers. The time interval between two-consecutive vector maps is $100 \mu \mathrm{s}$. Figure 3 shows sample images which are recorded at the speed of $20 \mathrm{kHz}$ and Reynolds number of 34,000 at the acute side. The obstacle is made of transparent acrylic resin and transmits the laser light. The diameter of the oil mist is about $10 \mu \mathrm{m}$ and the gap between the channel wall and the obstacle is $4000 \mu \mathrm{m}$. Since the ratio of the particle diameter to the gap is $1 / 400$, oil particle cannot be determined individually in these scales. However, the movements of mist pattern and dark vortices are seen very clearly.

Figure 4 shows time-serial relative velocity vector maps. In Figure 3, a vortex core is seen as a dark point because of centrifugal forces. Strong velocity fluctuations and vortex structures can be seen near to the boundary from the velocity vector maps.

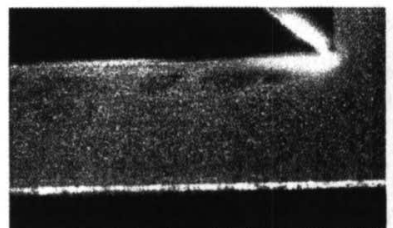

(a) $t=0 \mu \mathrm{s}$

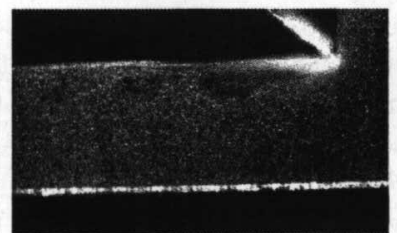

(b) $t=100 \mu \mathrm{s}$
Figure 3. Images for acute side $(\mathrm{Re}=34,000)$.

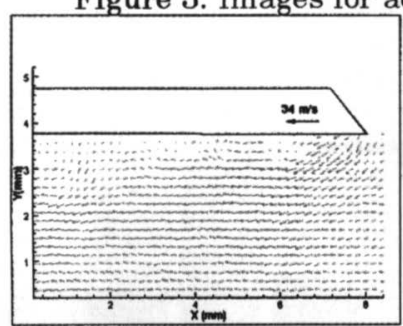

(a) $t=0 \mu \mathrm{s}$

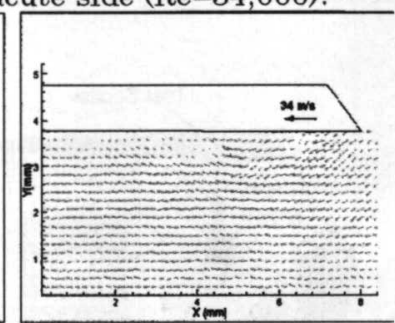

(b) $t=100 \mu \mathrm{s}$
Figure 4. Instantaneous relative velocity vectors.

\subsection{Two-point velocity cross-correlation and fluctuation transfer}

A measure of the degree of correlation between the two velocities at different spatial locations changes with respect to time. At the different spatial locations which are located on the downstream path of the vortexes, relatively huge and sudden velocity fluctuations are created. These velocity fluctuations, which are located inside the vortex structure, must have relations with each other's quantitatively.

The measure of the degree of correlation between the two velocity fluctuations can be defined as a function of space and time delay;

$$
C(x, y, \tau)_{k}=\frac{\int_{-\infty}^{\infty} u\left(x_{k}, y_{k}, t\right) u(x, y, t+\tau) d t}{\sqrt{\int_{-\infty}^{\infty} u\left(x_{k}, y_{k}, t\right)^{2} d t} \sqrt{\int_{-\infty}^{\infty} u(x, y, t+\tau)^{2} d t}}
$$

,where, $C(x, y, \tau)_{k}$ is the correlation value, a function of $x, y$, and time delay $\tau$. $k$ shows the chosen reference point index. $u(x, y, t)$ is the down-stream component of the fluctuation velocity.

The delay, $\tau$, is calculated for certain point $\left(x_{k} y_{k}\right)$. The gradient of the delay shows the transverse velocity of the fluctuations at the point.

$$
\frac{\partial \tau}{\partial x}=\frac{1}{u_{t r}}
$$

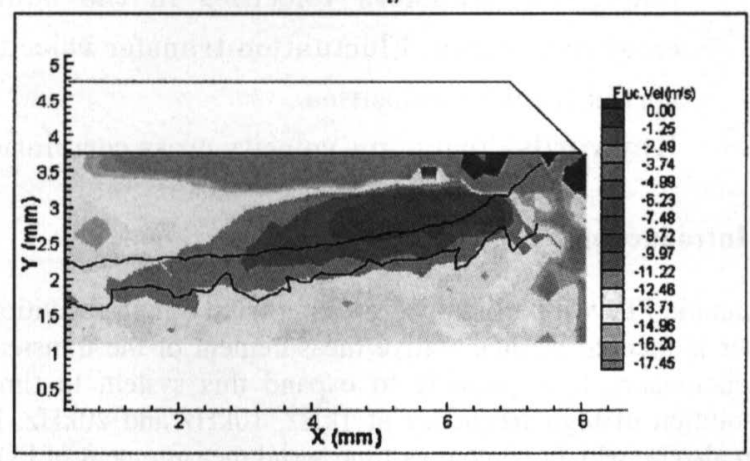

Figure 5. Fluctuation transfer velocity distribution in $\mathrm{m} / \mathrm{s}$. $(\mathrm{Re}=11000)$

Figure 5 shows the distributions of fluctuation velocity defined in Eq.(2). The fluctuation velocity near the edge region is smaller, while, it increases to the average velocity in downstream.

\section{Conclusions}

Dynamic PIV was applied to the high-speed mist flow, and then by using the PIV output velocity vectors spatial and temporal relations of the fluctuating velocities were analyzed by using the time dependent multi point velocity cross-correlation.

\section{Reference}

1) Report for Int. Workshop on Dynamic PIV 2004 Tokyo. 